\title{
HIRSTIONYSSUS GALEATUS, UN NOUVEAU GAMASIDE (ACARI) PARASITE DE MICROTUS ARVALIS LAEVIS
}

\author{
Par Zicman FEIDER et Libertina SOLOMON
}

On connaît une seule espèce du genre Hirstionyssus Fonseca 1948 (famille Liponyssidre Ewing 1923), H. isabellinus Oudemans 1913, qui parasite le campagnol (Microtus arvalis Pallas 1778). Nous décrivons dans la présente note une seconde espèce Hirstionyssus galeatus n. sp. Feider et Solomon, parasite sur une sous-espèce de campagnol Microtus arvalis laevis Miller 1912.

Nous avons examiné un nombre de onze femelles et une deutonymphe de notre espèce dont nous présentons la description.

\section{1) Femelle}

Face dorsale (fig. 1) : Le corps, long de 508-551 $\mu$ et large de 268-305 $\mu$, est ovale, allongé, un peu plus large à l'extrémité antérieure, où s'observent les épaules comme chez quelques autres espèces du genre [H. sciurinus (Hirst) 1921, H. carnifex (Koch) Oudemans 1913, H. georgicus Bregetova 1956, H. macedonicus Hirst 1921 et $H$. pauli Willmann 1952].

L'écusson dorsal, ayant les dimensions du corps, est recouvert par un lacis à mailles polygonales et 25 paires de poils de $12-29 \mu$. En dehors de l'écusson sont fixées 11 paires de poils de 11-26 $\mu$.

Face ventrale (fig. 2) : L'écusson sternal, long de $20 \mu$ et large de $110 \mu$, est très excavé sur le bord postérieur, ainsi qu'on l'observe chez $H$. isabellinus, $H$. meridianus Zemskaja 1955, H. eversmanni Zemskaja 1955 et $H$. transiliensis Bregetova 1956. L'écusson génitoventral, de $206 \mu$ de longueur et $145 \mu$ de largeur maximum, est très élargi à l'extrémité antérieure, où s'observent de fines stries, serrées et divergentes, ainsi qu'on l'observe chez $H$. confucianus (Hirst) 1921. Sa surface est divisée en six champs par plusieurs lignes ornementales. A l'endroit où l'écusson se rétrécit se trouve une paire de poils, de $32 \mu$, de même que chez $H$. blanchardi (Trouessart) 1904 et $H$. breviseta Strandtmann et Morlan 1953. 


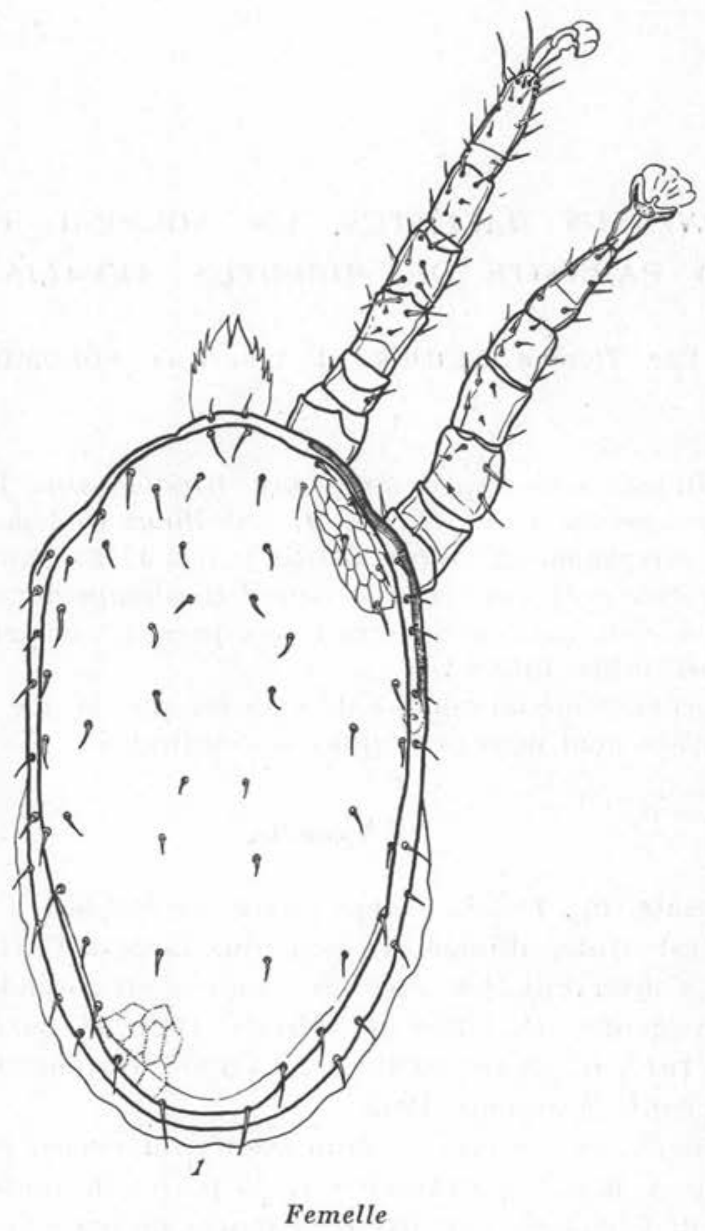

Fig. 1. - Corps, vue dorsale.

L'écusson anal, long de $99 \mu$ et large de $70 \mu$, est piriforme-ovalaire comme chez les espèces $H$. carnifex, $H$. isabellinus, $H$. talpæ (Zemskaja) 1954. La paire de poils anaux, de $23 \mu$, est fixée au niveau de la moitié postérieure des valves anales. La même position des poils anaux, paire, se trouve chez $H$. pauli, $H$. isabellinus, $H$. eversmanni, H. talpæ et $H$. musculi (Johnston) 1849.

$\mathrm{Au}$ niveau de la troisième paire de coxæ s'observent les poils métasternaux. Sur l'opisthosoma sont fixées 20 paires de poils, de 22 u. 


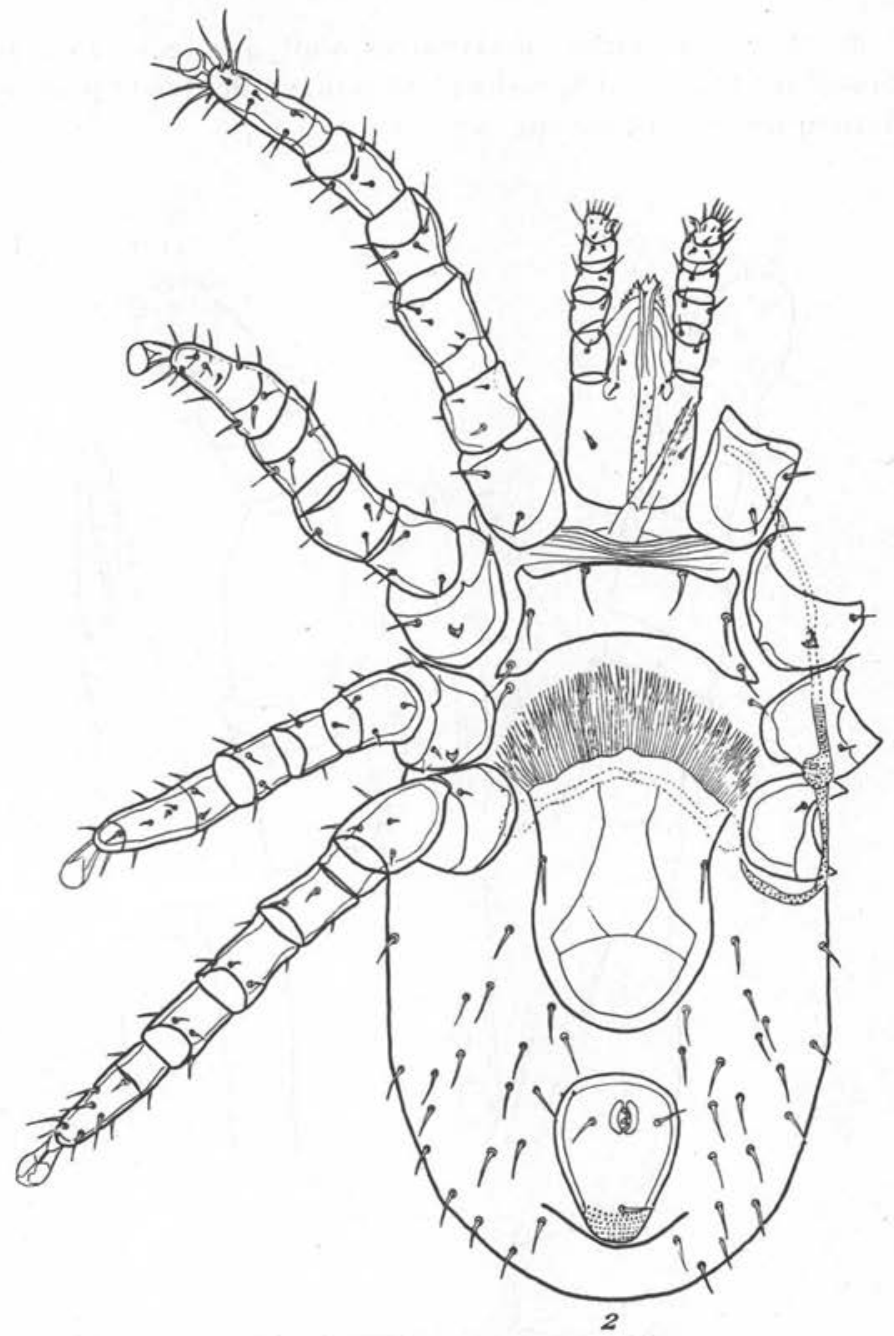

Fig. 2. - Corps, vue ventrale.

Les rameaux de bifurcation du tritosternum sont pourvus de poils sur les deux côtés.

Le péritrème, visible sur la face dorsale, s'étend antérieurement jusqu'au niveau de la première coxa et présente, à la partie postérieure, un appendice qui entoure la quatrième coxa.

Gnathosoma : Au-dessus du gnathosoma, qui est long de $148 \mu$ et large de $61 \mu$, se trouve un tectum dont le bord antérieur est pourvu de dix lambeaux, parmi lesquels les deux médians sont plus élargis 
(fig. 1, 3). Les cornicules maxillaires sont allongés. Les palpes maxillaires, de $116 \mu$, ont le palpetarse pourvu de douze poils et une griffe bifurquée et courbée (fig. 3).

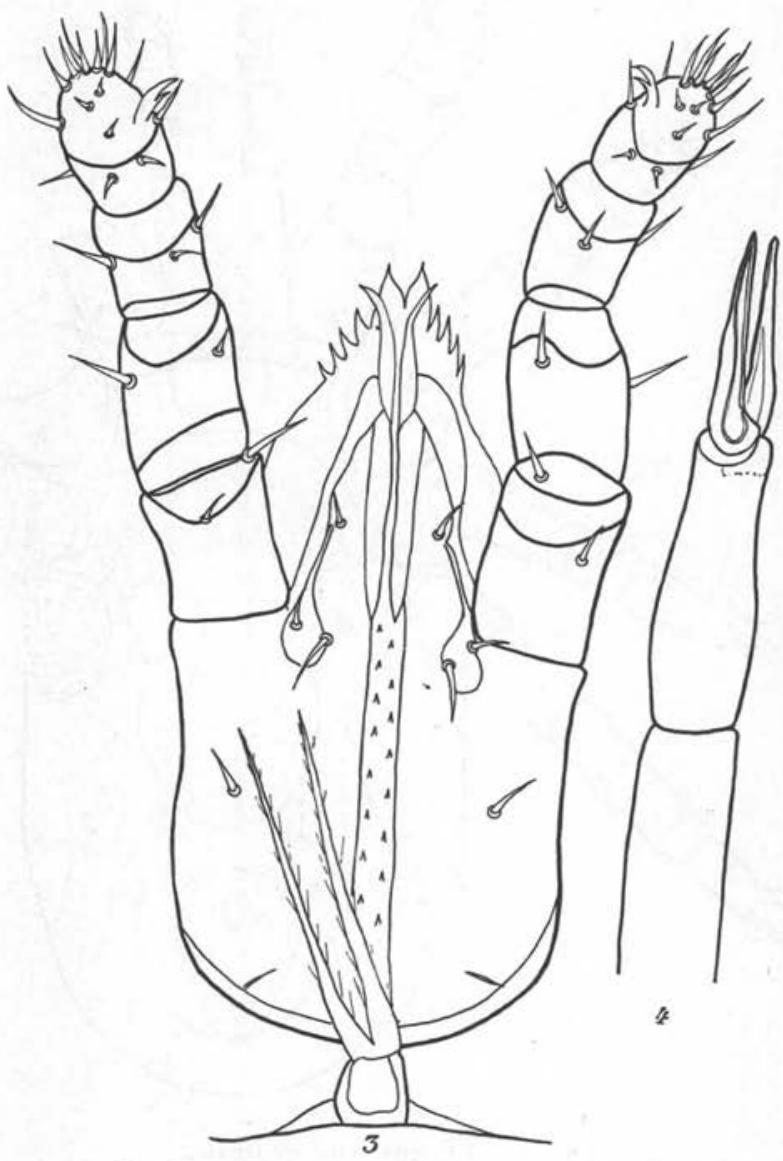

Fig. 3. - Gnathosoma, vue ventrale.

FIg. 4. - Chélicère.

Le chélicère, de $162 \mu$, a le digitus fixus de $41 \mu$ et le digitus mobilis de $46 \mu$. Les deux doigts s'effilent vers l'extrémité.

Pattes : La longueur des pattes est respectivement de $406 \mu, 348 \mu$, $319 \mu$ et $464 \mu$. La seconde et la troisième coxa sont pourvues d'un poil spécial dont l'extrémité épaissie a la forme d'un casque (d'où le nom galeatus). En outre, on trouve sur la seconde coxa une expansion triangulaire en forme d'épine associée avec un poil droit et, sur la troisième coxa, un seul poil droit. 


\section{2) Deutonymphe}

Face dorsale (fig. 5) : Le corps, long de $425 \mu$ et large de $261 \mu$, est plus large à la partie postérieure, en comparaison avec la femelle, et en même temps il a des épaules. L'écusson dorsal, long de $348 \mu$ et large de $174 \mu$, s'amincit vers les deux extrémités, spécialement vers l'extrémité postérieure. Chez $H$. bregetovæ Razumova 1953 et $H$. breviseta, l'extrémité postérieure de l'écusson est encore plus amincie.

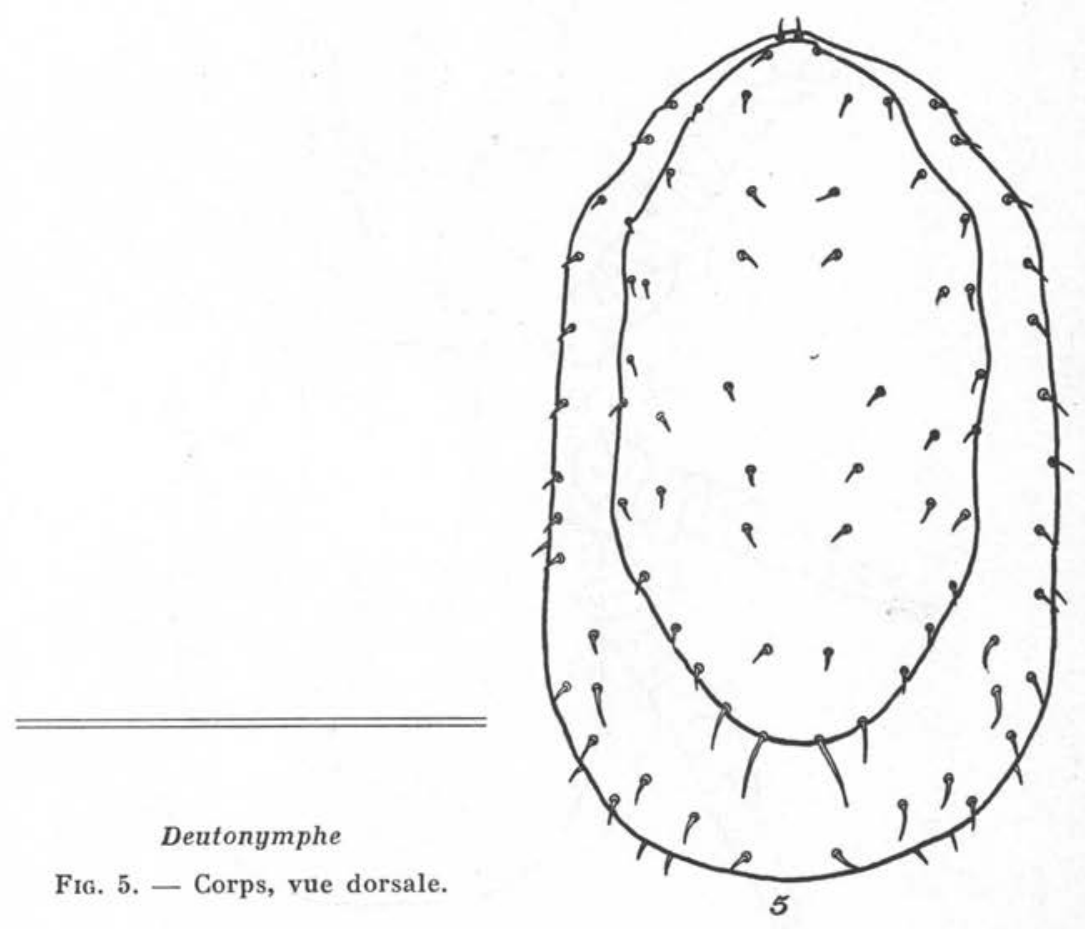

Sur la surface de l'écusson, outre le lacis à mailles polygonales, s'observent 23 paires de poils, de 6-9 $\mu$, à l'exception de la paire de poils pygidiaux, qui mesure $32 \mu$. En dehors de l'écusson, se trouvent 18 paires de poils, de 9-17 $\mu$ de longueur. Chez $H$. breviseta, la deutonymphe a un nombre moindre de poils.

Face ventrale (fig. 6) : Le sternum, long de $165 \mu$ et large de $96 \mu$, est triangulaire, avec les angles antérieurs aigus et les bords latéraux convexes, étant plus large que chez les espèces de comparai- 
son. Sur le bord du sternum sont fixées quatre paires de poils. L'écusson anal, long de $58 \mu$ et large de $38 \mu$, est ovalaire et plus allongé que chez la femelle. La paire de poils, de $9 \mu$, est fixée en avant du bord des valves anales. Le poil impair, de $7 \mu$, ne dépasse

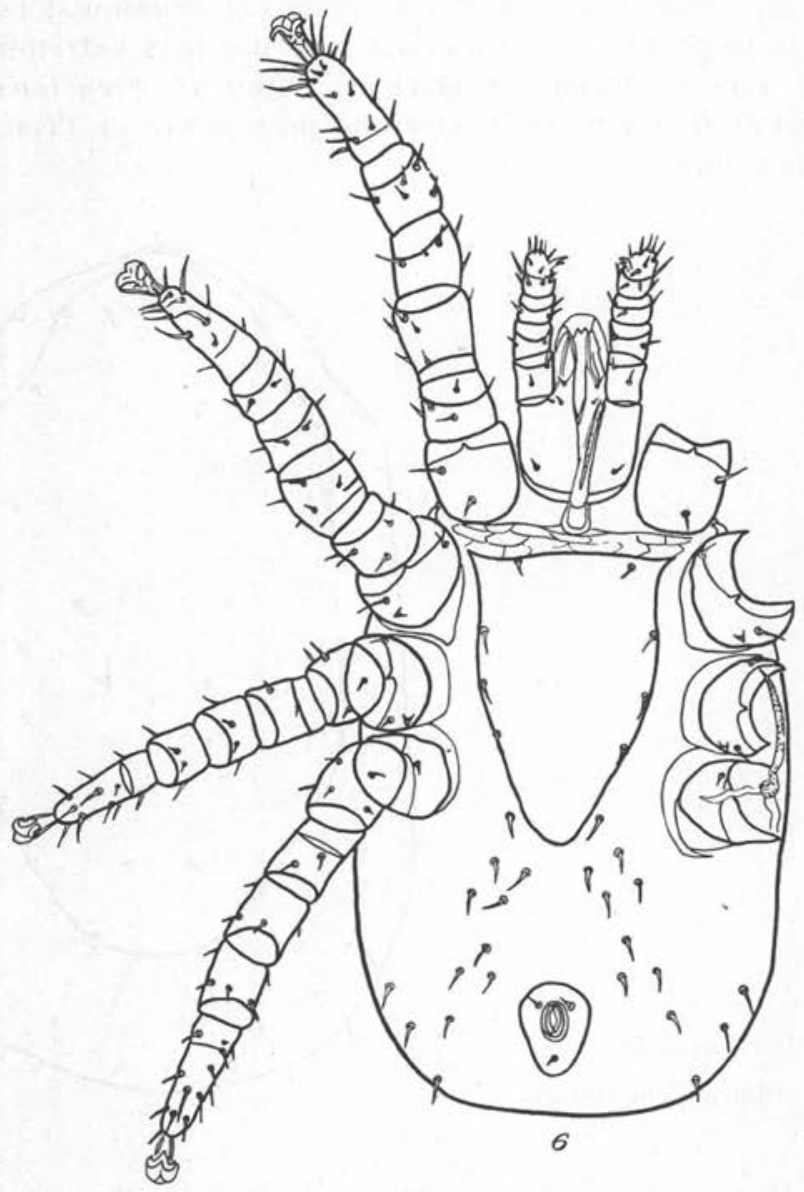

Fig. 6. - Corps, vue ventrale.

pas le bord postérieur de l'écusson. Les poils opisthosomaux, au nombre de douze paires, mesurent 9-12 $\mu$.

Le tritosternum ressemble à celui de la femelle, mais a un socle plus allongé.

Le péritrème arrive jusqu'au niveau de l'extrémité antérieure de la troisième coxa, tandis que, chez $H$. breviseta et $H$. bregetovæ, il 
s'étend plus antérieurement, le long de la seconde coxa. L'appendice postérieur du péritrème n'est pas développé.

Gnathosoma (fig. 7) : Le gnathosoma, long de $93 \mu$ et large de $67 \mu$, présente des cornicules maxillaires de la même forme que chez la femelle, mais avec des poils plus courts. Le bord du tectum est droit.

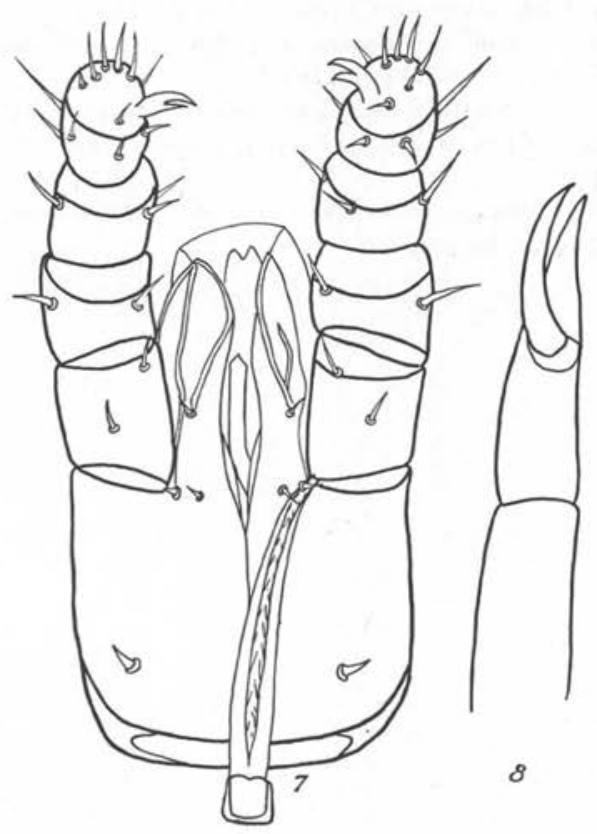

FIG. 7. - Gnathosoma, vue ventrale. FIG. 8. - Chélicère.

Les palpes maxillaires, de $90 \mu$, sont plus massives que chez la femelle. Le palpetarse est muni seulement de onze poils, mais la griffe a la même forme que chez la femelle.

Le chélicère, de $99 \mu$, a les deux doigts aigus mesurant $30 \mu$ (fig. 8).

Les pattes ont respectivement $305 \mu, 246 \mu, 246 \mu$ et $305 \mu$. Les coxæ de la seconde et de la troisième paire sont munies d'un poil spécial, mais plus aigu que chez la femelle. La seconde coxa présente en plus une épine triangulaire et un poil.

Les exemplaires de cette espèce ont été récoltés sur Microtus arvalis laevis, capturé sur la lisière d'une forêt, sur les bords de la rivière Pîngărati, à Pìngărati, le raion Bacău, les 2-10 juillet 1958.

Les types de l'espèce se trouvent chez les auteurs.

(Laboratoire de Zoologie, Université d'Issi, Roumanie). 


\section{Bibliographie}

1. Baker (E. W.), Ewans (T. M.), Gould (D. Y.), Hull (W. B.) and KeEgan (H. L.), 1956. - A manual of parasitic mites of medical or economic importance, New-York, Nat. Pest Control Assoc., Inc.

2. Bregetova (N. G.), 1956. - Gamazovîie klesei (Gamasoidea). Izd. Akad. Nauk., S.S.S.R., Moscva-Leningrad.

3. Kiyoshi Asanuma. - Studies on Ticks and Blood-sucking. Mites infesting Rats (genus Rattus) in Japan, Part. I, Research Inst. for Nat. Resources, Tokyo (Japan).

4. Vitzthum (H.). - Milben, Acari. Die Tierwelt Mitteleuropas, Verlag von Quelle et Meyer, Leipzig. 